\title{
Pig-hunting dogs are an at-risk population for canine heartworm (Dirofilaria immitis) infection in eastern Australia
}

Bronwyn Orr', Gemma Ma', Wei Ling Koh', Richard Malik², Jacqui M. Norris ${ }^{1}$, Mark E. Westman', Denise Wigney ${ }^{3}$, Graeme Brown ${ }^{1}$, Michael P. Ward ${ }^{1}$ and Jan Šlapeta ${ }^{1 *}$ (D)

\begin{abstract}
Background: Canine heartworm disease, caused by Dirofilaria immitis, has global veterinary importance. In Australia, the prevalence of canine heartworm infection decreased markedly following the introduction of over-the-counter macrocyclic lactones. We aimed to estimate the prevalence of canine heartworm infection in at-risk populations of dogs in eastern Australia and analyse published prevalence data from Australia.
\end{abstract}

Methods: In total, 566 dogs from eastern Australia were tested for the presence of $D$. immitis antigen. Four cohorts were studied: pig-hunting dogs from Queensland (Cohort 1, $n=104$ ), dogs from remote New South Wales (NSW) (Cohort 2, $n=332$ ), urban pets from rural NSW (Cohort $3, n=45$ ) and ex-racing Greyhounds from Sydney, NSW (Cohort $4, n=85$ ). Serum samples were screened for $D$. immitis antigen using a reference laboratory microwell-based assay $\left(\right.$ DiroChek ${ }^{\circledR}$ ) or a point-of-care immunochromatography test kit (Anigen Rapid ${ }^{\circledR}$ ). Risk factors associated with the odds of D. immitis antigen seropositivity were identified using binary logistic regression models. Seropositive blood samples were tested for the presence and quantity of D. immitis DNA using a species specific real-time (q)PCR assay. A metanalysis of the Australian canine heartworm literature was conducted.

Results: The prevalence of dirofilariasis in pig-hunting dogs from Queensland (Cohort 1) was 12.5\% (95\% Cl: 6.518.9\%), with a subpopulation of dogs from Central Queensland having a prevalence of 21\% (95\% Cl: 12.3-33.4\%). Age was significantly associated with D. immitis antigen seropositivity (increased risk with increased age). The odds of being $>5$ years versus $\leq 5$ years was 3.7 -times ( $95 \%$ Cl: $1.1-12.5)$ greater in antigen positive versus antigen negative dogs. No D. immitis antigen positive dogs were detected in dogs from NSW (Cohorts 2-4). The Australian canine heartworm disease literature includes 98 peer-reviewed publications (1901-2019) with 30 studies reporting on D. immitis prevalence in dogs. Throughout the publication peak period (1980s), the primary antemortem diagnostic test was detection of microfilariae.

Conclusions: Canine heartworm infection in dogs used for pig hunting is a previously unexplored topic in Australia. Pig-hunting dogs are infected with canine heartworm in Queensland, Australia, placing pet dogs and cats at increased risk of infection.

Keywords: Heartworm, Dirofilaria immitis, Canine, Pig-hunting, Australia, Prevalence, At-risk population

*Correspondence: jan.slapeta@sydney.edu.au

1 Sydney School of Veterinary Science, The University of Sydney, Sydney, NSW 2006, Australia

Full list of author information is available at the end of the article

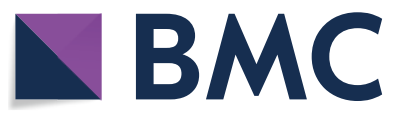

(c) The Author(s) 2020. This article is licensed under a Creative Commons Attribution 4.0 International License, which permits use, sharing, adaptation, distribution and reproduction in any medium or format, as long as you give appropriate credit to the original author(s) and the source, provide a link to the Creative Commons licence, and indicate if changes were made. The images or other third party material in this article are included in the article's Creative Commons licence, unless indicated otherwise in a credit line to the material. If material is not included in the article's Creative Commons licence and your intended use is not permitted by statutory regulation or exceeds the permitted use, you will need to obtain permission directly from the copyright holder. To view a copy of this licence, visit http://creativeco mmons.org/licenses/by/4.0/. The Creative Commons Public Domain Dedication waiver (http://creativecommons.org/publicdomain/ zero/1.0/) applies to the data made available in this article, unless otherwise stated in a credit line to the data. 


\section{Background}

Canine heartworm disease (dirofilariasis) is caused by the filarial nematode Dirofilaria immitis [1, 2]. Left untreated, canine heartworm disease can become lifethreatening, with the parasite's tropism for the pulmonary arterial vasculature causing inflammation, thrombo-embolism, pulmonary hypertension and eventually, right-sided heart failure [3, 4].

Historically, Australia experienced high rates of canine heartworm disease across several regions in the 1970s and 1980s. During this era, the prevalence of canine heartworm in Queensland, Northern Territory and New South Wales (NSW) was reported to range from 30\% to $100 \%$, with the tendency for higher prevalence to occur in the tropical and subtropical regions of northern Australia [5-10]. Since then, the prevalence of canine heartworm in Australia has decreased progressively. By the early 1990s, a wide range of once-a-month oral or 'spot-on' macrocyclic lactones (MLs) for canine heartworm prevention had become available in Australia. These preventatives did not require a prescription and could be purchased across the counter from veterinary clinics, pet stores and (later) from online retail outlets and supermarkets [11, 12]. In 2016, despite these measures, an apparent re-emergence of $D$. immitis infection and canine heartworm disease was documented in pet dogs in Central Queensland [13]. Apart from the impact of prophylactic therapy, factors affecting the prevalence of canine heartworm disease in Australia are incompletely understood.

For D. immitis to successfully (re)emerge and sustain its life-cycle, certain pre-requisites need to be met. First, the parasite is mosquito-borne. In Australia, the competent vectors have been shown to be mosquitoes of the genera Aedes, Culex and Anopheles [10, 14, 15]. Secondly, there needs to be an absence of prophylactic treatment with MLs, either through poor or absent client compliance or inadequate dosing, as this probably represents the most common reason for canine heartworm infection globally [16-18]. Finally, there needs to be competent species acting as reservoirs for $D$. immitis such as wild dogs, dingoes and owned dogs not receiving prophylaxis [19]. Identifying and monitoring canine cohorts that do not receive regular heartworm prevention (at-risk populations) is imperative, particularly in regions with low endemicity. One possible group in Australia is pig-hunting dogs. Hunting feral pigs (known as hogs in the USA) is a popular activity in Australia, with an estimated 156,000 dogs kept specifically for this purpose [20] (Fig. 1). Recent enquiries into the health and welfare of pig-hunting dogs in Australia and New Zealand suggested they were at high risk of exposure to numerous infectious diseases and received minimal veterinary preventive care $[20,21]$.
In this study, we aimed to identify at-risk populations of dogs based on a history of incomplete canine heartworm (D. immitis) prevention. To do so, we used $D$. immitis antigen testing and positive samples were tested for the presence of D. immitis DNA. Four populations of dogs with moderate or no canine heartworm prevention were tested, including pig-hunting dogs, remote community dogs, owned urban dogs and ex-racing Greyhounds. For the cohort of pig-hunting dogs, reference laboratory $D$. immitis antigen testing was performed to evaluate the accuracy of a point-of-care immunomigration $D$. immitis antigen test. The context of canine heartworm in Australia is explained using a historical review (metanalysis) of published data.

\section{Methods \\ Dog cohorts from Queensland and New South Wales, Australia}

Four canine cohorts in Australia were sampled to determine the presence of canine heartworm (D. immitis) antigen (Fig. 2, Table 1). In total, 566 dogs were sampled. Whole blood samples were separated into serum and stored at $-20{ }^{\circ} \mathrm{C}$ prior to testing at the Sydney School of Veterinary Science (SSVS), University of Sydney.

Cohort 1 [Pig-hunting dogs $(n=104)$ ] were sampled from August to November 2018 from towns across Central and North Queensland, Australia. Locations included Sarina, Clermont, Proserpine, Charters Towers, Innisfail, Tully, Malanda and Atherton. Dogs sampled had been used for hunting feral pigs (hogs), and generally were large, mixed breed dogs living outdoors in kennels or yards. Based on currently unpublished survey data from the owners, only intermittent canine heartworm prophylaxis was used, if at all.

Cohort 2 [Remote community dogs $(n=332)$ ], predominantly crossbreeds, were sampled from six communities across remote and very remote western New South Wales between 2016-2018. Dogs were recruited from the participants of Royal Society for the Prevention of Cruelty to Animals (RSPCA) NSW Community Companion Animal Health Programs, a service provided for people on low incomes, many of whom identify as Aboriginal. These dogs have limited or no access to veterinary treatment including canine heartworm prophylaxis.

Cohort 3 [Owned urban dogs $(n=45)$ ] were sampled between June and September 2018 in Goulburn, a small inland city in NSW, Australia. These dogs were all pets visiting a local veterinary clinic, none of which were receiving heartworm prophylaxis from their owners at the time of sampling.

Cohort 4 [Adopted ex-racing Greyhounds $(n=85)$ ] were sampled between January 2018 and November 2019 


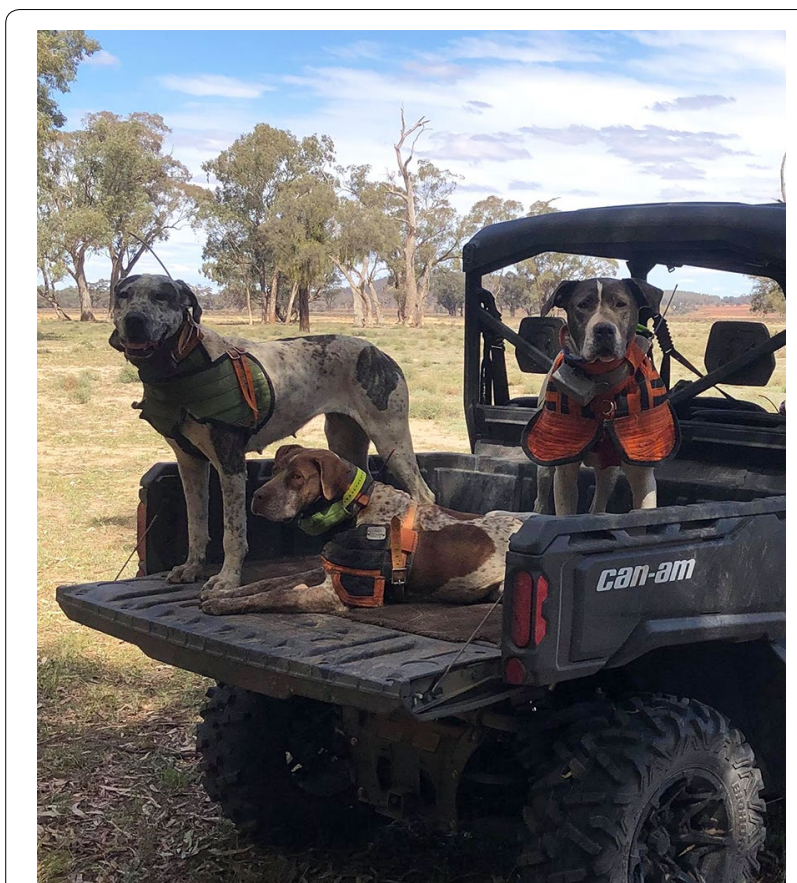

Fig. 1 Pig-hunting dogs in Australia. Image courtesy of John Calvani

in the Greater Sydney Region, NSW. These dogs were all part of Greyhound Adoption Program (NSW) Inc, having retired from racing and currently living as pet dogs. More than half of these dogs $(46 / 85,54 \%$; self-reported by owners) were receiving monthly heartworm preventatives, although their treatment history was not known in any more detail.

\section{Testing for Dirofilaria immitis antigen}

Serum samples from Cohorts 1-3 were tested using DiroChek ${ }^{\circledR}$ Heartworm Antigen Test Kits (Zoetis, Parsippany, USA), a qualitative microwell-based enzyme-linked immunosorbent assay (ELISA) for the detection of adult female $D$. immitis antigen. DiroChek ${ }^{\circledR}$ is intended for use by diagnostic laboratories and was used according to manufacturer's instructions; 5 min after the addition of the final solution, the 96-well plate or 8-well strip with manufacturers positive and negative controls were visually examined for a clear to blue color change. All blue colored wells, regardless of intensity, were considered antigen positive or, if no blue color was detected, samples were considered to have 'no detectable antigen' (NDA).

Serum samples from Cohorts 1 and 4 were tested using point-of-care Anigen Rapid $^{\circledR}$ Canine Heartworm (CHW) Ag 2.0 Test Kits (BioNote, Gyeonggi-do, Korea) a chromatographic immunoassay for the detection of D. immitis antigen. Sensitivity and specificity of Anigen
Rapid $^{\circledR}$ CHW Ag 2.0 for D. immitis antigen have been reported as $99.5 \%$ and $94 \%$, respectively [22]. The Ani-

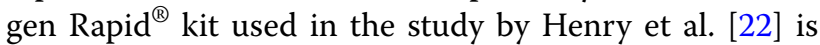
identical to the one marketed and sold in Australia (Mark Thacker, Life Bioscience, Oakleigh, Australia, personal communication).

Knott's test for the identification of D. immitis microfilariae (Mff) was not performed due to the logistical constrains, remote sampling locations and lack of laboratory resources in the field.

\section{Dirofilaria immitis specific real-time PCR}

Blood clot samples $(200 \mu \mathrm{l})$ from $D$. immitis antigen positive dogs and a random selection of NDA $D$. immitis dogs (Cohort 1) were used for molecular studies. Genomic DNA was isolated using PowerMag ${ }^{\circledR}$ Blood DNA/RNA Isolation Kits (Qiagen, Chadstone, Australia) optimised for the KingFisher ${ }^{\circledR}$ Duo Prime Purification System (Thermo-Fisher Scientific, Scoresby, Australia). DNA isolation was performed as a batch of 11 blood samples and an extraction blank. DNA eluted in $100 \mu \mathrm{l}$ was stored in aliquots at $-20{ }^{\circ} \mathrm{C}$ prior to analysis.

Presence of canine DNA was verified using real-time PCR (qPCR) to amplify partial canine glyceraldehyde3-phosphate dehydrogenase (GAPDH), forward primer (S0631): 5'-TCA ACG GAT TTG GCC GTA TTG G-3' and reverse primer (S0634): 5'-TGA AGG GGT CAT TGA TGG CG-3' with probe (S0632): 5'-HEX-CAG GGC TGC TTT TAA CTC TGG CAA AGT GGABHQ1-3'; RTPrimerDB ID: 1193 [23]. PCR cycling conditions included an initial step at $95{ }^{\circ} \mathrm{C}$ for 3 min followed by 40 two-step cycles of $95{ }^{\circ} \mathrm{C}$ for $5 \mathrm{~s}$ and $60{ }^{\circ} \mathrm{C}$ for $15 \mathrm{~s}$. The final qPCR reaction mixture $(20 \mu \mathrm{l})$ included $10 \mu \mathrm{l}$ of SsoAdvanced Universal Probes Supermix (BioRad, Gladesville, Australia), each primer at $400 \mathrm{nM}$ concentrations, probe at $100 \mathrm{nM}$ concentration, PCR-grade water, and $2 \mu \mathrm{l}$ of DNA template. PCR testing was performed using a CFX96 Touch $^{\mathrm{TM}}$ Real-Time PCR Detection System with the corresponding CFX Manager v.3.1 software (BioRad, Gladesville, Australia). The arbitrary qPCR threshold was determined automatically using default settings and threshold cycle $\left(\mathrm{C}_{\mathrm{q}}\right)$-values reported.

A species-specific $D$. immitis qPCR assay and microfilariae (Mff) quantification was performed, as described [13]. Primers targeting the cox 1 gene fragment ( $203 \mathrm{bp})$ of $D$. immitis were used, consisting of forward primer (S0582): 5'-AGT GTA GAG GGT CAG CCT GAG TTA-3' and reverse primer (S0583): 5'-ACA GGC ACT GAC AAT ACC AAT-3) [24]. The final qPCR reaction mixture $(20 \mu \mathrm{l})$ included $10 \mu \mathrm{l}$ of SsoAdvanced Universal SYBR $^{\circledR}$ Green Supermix (BioRad, Gladesville, Australia), each primer at $400 \mathrm{nM}$ concentrations, PCR-grade water, and $2 \mu \mathrm{l}$ of DNA template. Each sample was run 



Fig. 2 Dogs surveyed for the presence of Dirofilaria immitis antigen in Australia. a Geographical distribution of dogs tested for heartworm as part of 2016-2019 prevalence surveys. The size of the circle at each location in New South Wales and Queensland (Central and Northern) is proportional to the number of tested dogs, number in brackets indicates number of D. immitis antigen positive dogs / total number of tested dogs. $\mathbf{b}$ Violin plots of distribution of age of 566 dogs sampled as part of four distinct cohorts, mean and quartiles are show within the violins. On the far left of the figure is a scatter dot plot of the individual ages of 13 antigen-positive dogs

Table 1 Summary of 566 dogs tested for Dirofilaria immitis antigen across eastern Australia 2016-2019

\begin{tabular}{|c|c|c|c|c|c|}
\hline \multirow[t]{2}{*}{ Cohort } & \multirow[t]{2}{*}{ Locality } & \multirow[t]{2}{*}{$\mathrm{Age}^{\mathrm{a}}$} & \multicolumn{2}{|c|}{ Positive/total } & \multirow[t]{2}{*}{ Canine heartworm prophylaxis } \\
\hline & & & DiroChek $^{\circledR}$ & Anigen Rapid ${ }^{\circledR}$ & \\
\hline \multicolumn{6}{|l|}{ Queensland } \\
\hline \multirow[t]{2}{*}{ Cohort 1} & Northern & $3(0.1-13)$ & $1 \mathrm{~b} / 42$ & $1 \mathrm{~b} / 42$ & None or intermittent \\
\hline & Central & $3(0.1-9)$ & $12 / 62$ & $11 / 62$ & None or intermittent \\
\hline \multicolumn{6}{|c|}{ New South Wales } \\
\hline Cohort 2 & Remote western & $1(0.5-12)$ & $0 / 332$ & nd & None \\
\hline Cohort 3 & Goulburn & $9(1-15)$ & $0 / 45$ & nd & None \\
\hline Cohort 4 & Greater Sydney & $5(2-9)$ & nd & $0 / 85$ & Rigorous in $54 \%(46 / 85)$ \\
\hline
\end{tabular}

\footnotetext{
${ }^{a}$ Median (minimum-maximum), in years

${ }^{b}$ A 9-year-old male relocated from Central Queensland

Abbreviations: nd, not determined; DiroChek ${ }^{\circledR}$, DiroChek ${ }^{\circledR}$ Heartworm Antigen Test Kit (Zoetis, NJ); Anigen Rapid ${ }^{\circledR}$, Rapid Test Kit Heartworm (CHW) Ag2.0 (Anigen, BioNote Inc., Seoul, South Korea)
}

in duplicate with 10 -fold serial dilutions $\left(1.96 \times 10^{2}\right.$ to $1.96 \times 10^{7}$ copies per reaction) of a known concentration of plasmid DNA containing the D. immitis cox 1 gene synthetised by GeneArt (Thermo Fisher Scientific, Scoresby,
Australia) and with negative (no template) controls, as described [13]. Amplification was performed as follows: initial denaturing step at $95{ }^{\circ} \mathrm{C}$ for $3 \mathrm{~min}$, followed by 40 cycles of denaturing $\left(5 \mathrm{~s}\right.$ at $\left.95^{\circ} \mathrm{C}\right)$ and annealing 
$\left(15 \mathrm{~s}\right.$ at $\left.62{ }^{\circ} \mathrm{C}\right)$. A final melting curve was produced by heating the product from $60{ }^{\circ} \mathrm{C}$ to $90{ }^{\circ} \mathrm{C}$ for $5 \mathrm{~s}$ at $0.5^{\circ} \mathrm{C}$ increments. PCR testing was performed using a CFX 95 Touch $^{\text {TM }}$ Real-Time PCR Detection System (BioRad Laboratories, Gladesville, Australia). $\mathrm{C}_{\mathrm{q}}$ values and standard curves were determined CFX Manager ${ }^{\mathrm{TM}}$ Software v. 3.1 (BioRad Laboratories, Gladesville, Australia). Any sample that amplified with a $\mathrm{C}_{\mathrm{q}}$-value $<40$ was sent for DNA purification and sequencing using amplification primers (Macrogen Inc., Seoul, Korea). Sequence chromatographs were manually inspected and compared to a reference $D$. immitis cox1 sequence (NC_005305) using CLC Main Workbench 6.9.1. (CLC Bio, Qiagen, Chadstone, Australia).

\section{Review of published canine heartworm prevalence data in Australia}

Using the University of Sydney's Library Search engine, in August 2019 we searched the peer-reviewed literature for the following terms: 'heartworm AND Australia' (789 results), 'Dirofilaria immitis AND Australia' (793 results) and 'dirofilariasis AND Australia' (275 results), with no restriction on material type and date and the search terms located in 'any field' within each database entry. The Library Search engine searches across library resources, including the library catalogue and journal databases such as Medline, Web of Science and Scopus. In addition, we conducted a second search in the Australian Veterinary Journal archives using the following terms: 'heartworm', 'Dirofilaria immitis' and 'dirofilariasis'. We examined peer-reviewed references from a specific $\mathrm{PhD}$ thesis (Martin TE, 1986: 'Prevalence and diagnosis of infection with Dirofilaria immitis in dogs', University of Sydney) as well as reference lists from all reviewed articles. Once collated, retrieved articles were manually inspected to confirm the presence of heartworm prevalence data and then reviewed in more detail. Inclusion criteria for retained references included primary data on D. immitis prevalence in dogs, regardless of the method used. For each retained reference, information on the year of study, study area, sample size, number of samples positive for D. immitis and test used was analysed.

\section{Statistical analysis}

Confidence intervals $(95 \% \mathrm{CI})$ were calculated using the modified Wald method. For Cohort 1, the association between heartworm antigen status (positive, negative) and age ( $\leq 5$ years versus $>5$ years), type ('Bull Arab' or 'Bull Arab crossbreed' versus others), sex (male versus female) and location (inland versus coastal) was tested using binary logistic regression models $(P<0.05)$. Possible associations were assessed using odds ratios $\left(\right.$ IBM $^{\circledR}$ SPSS Statistics v24). Non-parametric Kruskal-Wallis one-way ANOVA testing with Dunn's multiple post hoc comparisons was used for age of cohorts because of the non-normal age distribution within each cohort (GraphPad Prism 8.2.1). The sensitivity and specificity of Anigen Rapid $^{\circledR}$ were calculated using results from Cohort 1 and the DiroChek ${ }^{\circledR}$ result as the gold standard, and the degree of agreement between Anigen Rapid ${ }^{\circledR}$ and DiroChek ${ }^{\circledR}$ quantified by determining the Kappa statistic (GraphPad Prism 8.2.1).

\section{Results}

Of the 566 dogs tested for D. immitis antigen, 13/566 (2.3\%; 95\% CI: $1.3-3.9 \%)$ were positive (Table 1, Fig. 2a). All 13 antigen positive results were from pig-hunting dogs in Queensland (Cohort 1), comprised of nine males and four females (13/104; 13\%; 95\% CI: 6.5-18.9\%). All but one $D$. immitis antigen positive dogs came from Sarina, Proserpine or Clermont (the Mackay and Whitsundays region of Central Queensland). Considering this region alone, $12 / 57$ dogs tested antigen positive $(21 \%$, 95\% CI: $12.3-33.4 \%)$. The remaining D. immitis antigen positive dog came from Malanda (Atherton Tablelands region of Far North Queensland), although this dog had been imported from Central Queensland.

No dogs tested in NSW (Cohorts 2-4, $n=462$ ) returned a positive antigen test result and all were therefore considered NDA. The age distribution of dogs was significantly different between the four cohorts (KruskalWallis one-way ANOVA, $H=237.1, d f=3, P<0.0001$ ), with all cohorts being significantly different from each other (Dunn's multiple comparisons, $P<0.05$ ). The median age of Cohort 2 was the lowest, followed by Cohorts 3, 1 and 4 (Fig. 2b, Table 1).

In Cohort 1 , antigen status was significantly $(P=0.03)$ associated with age: the odds of being $>5$ years versus $\leq 5$ years was 3.73-times (95\% CI: $1.1-12.5$ ) greater in seropositive versus seronegative dogs (binary logistic regression models). Seropositivity was not associated with sex $(P=0.37$; male versus female) or type $(P=0.6$; Bull Arab and crossbreds versus others). Location $(n=8)$ as a fixed effect was not significantly $(P=0.54)$ associated with antigen status. However, location categorised as coastal versus inland was significantly $(P=0.03)$ associated with serostatus: the odds of being coastal versus inland was 3.89-times (95\% CI: 1.2-12.8) higher in antigen positive versus antigen negative dogs. Finally, both age and location were significantly associated with serostatus when included in the same logistic regression model (Table 2). This model adequately fitted the data (Hosmer \& Lemeshow test, $\chi^{2}=1082, d f=2, P=0.58$ ), while the interaction between age and location was not significant $(P=0.30)$. 
The sensitivity of the Anigen Rapid ${ }^{\circledR}$ test was 92\% (95\% CI: 62.1-99.6\%) and specificity was 100\% (95\% CI: 95.1-100\%), while the kappa statistic was 0.96 (95\% CI: 0.87-1.00) (Table 3).

To determine if the $D$. immitis antigen positive pighunting dogs were a source of $D$. immitis for mosquitoes (i.e. that they were microfilaraemic), we used available blood clots from 11/13 antigen positive dogs and a selection $(n=9)$ of antigen-negative dogs (Table 3). DNA was successfully purified and canine GAPDH amplified $\left(\mathrm{C}_{\mathrm{q}}\right.$ values: 21.8-31.5) from 20 canine blood samples. Blank DNA samples remained negative in the canine GAPDH assay. Seven Cohort 1 samples $(7 / 11,64 \%)$ from $D$. immitis antigen positive dogs were considered positive for D. immitis microfilariae DNA (Table 3). The calculated microfilariae concentration ranged from $<1$ to 22,050 microfilariae/ml (Table 3). Four of 11 samples returned negative results $(0$ to $<5)$ for $D$. immitis DNA for samples that were $D$. immitis antigen positive (Table 3 ). These negative results are consistent with a concentration of microfilariae $<5 / \mathrm{ml}$ of blood, because we used $200 \mu \mathrm{l}$ of blood for DNA isolation and thus required at least 1 microfilaria for a positive DNA result. All antigennegative samples $(n=9)$ yielded negative results for the presence of $D$. immitis microfilariae DNA (Table 3), as expected. For the $D$. immitis DNA positive samples, DNA sequencing of the cox 1 PCR amplicon was $100 \%$ identical with the D. immitis cox 1 gene reference sequence (GenBank: NC_005305).

Searches to determine the historic prevalence of canine heartworm disease in Australia yielded 98 peer-reviewed publications spanning the years 1901-2019 (Figure 3). After applying our inclusion criteria, 32 studies remained (Figure 3, Table 4). Of these, 30 studies reported on $D$. immitis prevalence in dogs, and two studies included data on D. immitis prevalence in red foxes (Table 4). Most publications reporting $D$. immitis in canids were published during 1966-2005, with a peak of 12 publications during the 1980s (Figure 3). Throughout this peak period, the primary antemortem diagnostic test was detection of microfilariae (Table 4).

\section{Discussion}

The only canine heartworm (D. immitis) antigen positive dogs detected in our survey of 566 dogs were pig-hunting dogs from Queensland. All 13 D. immitis antigen-positive dogs, including one domiciled in Far North Queensland at the time of testing, had spent time in the Mackay and Whitsundays regions of Central Queensland. Recent research by Nguyen et al. [13] demonstrated the presence of $D$. immitis in this region in dogs visiting veterinary practices. More than one-fifth of the pig-hunting dogs tested in Central Queensland were D. immitis antigen-positive. The current prevalence of $D$. immitis antigen seropositivity in pig-hunting dogs in this region is comparable to the historical $D$. immitis prevalence data in pound dogs based on necropsy or microfilaraemia in Queensland during 1960-1990 [6, 7, 10, 25]. A significantly higher prevalence of $D$. immitis antigen seropositivity in dogs older than five years of age compared to

Table 3 Summary of molecular confirmation of Dirofilaria immitis using species specific qPCR

\begin{tabular}{lllll}
\hline Sample ID & DiroChek $^{\circledR a}$ & Anigen Rapid $^{\circledR b}$ & $\begin{array}{l}\text { Estimated D. } \\
\text { immitis (Mff. } \\
\mathrm{ml}^{-1} \text { ) }\end{array}$ & $\begin{array}{l}\text { Host (dog) } \\
\mathrm{C}_{\mathrm{q}}(\mathrm{GAPDH})\end{array}$ \\
\hline BO\#231 & Positive & Positive & $<5$ & 26.17 \\
BO\#103 & Positive & Positive & $<5$ & 24.54 \\
BO\#135 & Positive & NDA & 503 & 23.76 \\
BO\#102 & Positive & Positive & 537 & 25.38 \\
BO\#55 & Positive & Positive & 6591 & 28.13 \\
BO\#56 & Positive & Positive & 7665 & 27.13 \\
BO\#138 & Positive & Positive & 22,050 & 24.34 \\
BO\#134 & Positive & Positive & 0 & 23.81 \\
BO\#136 & Positive & Positive & 0 & 24.71 \\
BO\#168 & Positive & Positive & 0 & 26.61 \\
BO\#58 & Positive & Positive & 0 & 27.23 \\
\hline
\end{tabular}

${ }^{a}$ DiroChek ${ }^{\circledR}$ Heartworm Antigen Test Kit (Zoetis, NJ)

${ }^{\text {b }}$ Anigen Rapid ${ }^{\circledR}$ Test Kit Heartworm (CHW) Ag2.0 (Anigen, BioNote Inc., Seoul, South Korea)

Abbreviations: NDA, no detectable antigen; Mff, D. immitis microfilariae

Table 2 Risk factors associated with the odds of Dirofilaria immitis antigen seropositivity of 104 pig-hunting dogs in North Queensland, Australia

\begin{tabular}{lllllll}
\hline Variable & Category & Beta & SE & P-value & Odds ratio & $95 \% \mathrm{Cl}$ \\
\hline Age & $\leq 5$ years & 0 & - & - & 1 & - \\
& $>5$ years & 1.331 & 0.642 & 0.038 & 3.786 & $1.076-13.326$ \\
Location & Inland & 0 & - & - & 1 & - \\
& Coastal & 1.371 & 0.629 & 0.029 & 3.940 & $1.148-13.522$ \\
\hline
\end{tabular}

Abbreviations: SE, standard error; $95 \% \mathrm{Cl}, 95 \%$ confidence interval 
younger dogs has previously been reported in Australia, and is a consequence of increased likelihood of infection with the cumulative exposure to more mosquito bites throughout life [6, 26]. In a large Australian study [10], no dog younger than 9 months showed presence of D. immitis microfilariae, and male dogs were more often positive then females. Possibly due to smaller samples sizes of atrisk dogs in our study, there was no difference observed in the sex of the dog in relation to antigen seropositivity.

Pig-hunting dogs are thought to receive minimal veterinary and preventative health care and often travel widely with their owners during hunting expeditions [20, 21]. Pig-hunting dogs are generally robust, large breed, shortcoated dogs that live outdoors [20], increasing their risk of being bitten by mosquitoes and thereby developing canine heartworm disease. Since they often reside close to pet dog populations, it will be important to investigate their potential as a reservoir for pet dogs sharing the same geography and receiving incomplete or lax canine heartworm preventative therapy.

In this study, we principally used the reference laboratory test, DiroChek ${ }^{\circledR}$ Heartworm Antigen Test Kit, for detection of adult D. immitis antigen. Courtney et al. [27] reported DiroChek ${ }^{\circledR}$ sensitivity to be $86 \%$ and specificity
97\% in the USA. More recently, Henry et al. [22] re-evaluated the accuracy of DiroChek ${ }^{\circledR}$ testing in the USA using a cohort of dogs with known $D$. immitis status, reporting test sensitivity of 99\% (95\% CI: 96.4-99.9\%) and specificity of $96 \%$ (95\% CI: 86.3-99.5\%), including a cohort of 50 dogs with low worm burdens (1-5 female $D$. immitis; Mff in $58 \%$ of dogs). In Australia, the DiroChek ${ }^{\circledR}$ has been tested extensively for its ability to detect $D$. immitis during the prepatent period and low heartworm burdens [5, 28, 29]. The DiroChek ${ }^{\circledR}$ was validated in 1987 by testing 100 dogs from Sydney, NSW and immediately performing necropsy examination, with the test correctly identifying $15 / 24$ infected dogs (sensitivity $62.5 \%$ ) and 74/76 uninfected dogs (specificity 97.4\%) [29]. Dogs returning a false negative result using DiroChek ${ }^{\circledR}$ included four dogs with one male heartworm, three dogs with two male worms, one dog with one male and two female worms and one dog with five male worms [29]. In another group of 100 pound dogs from Sydney examined by necropsy, 8 dogs that were negative using DiroChek ${ }^{\circledR}$ had adult heartworms at necropsy, four with prepatent infections and four with infections of three or less worms, all of one sex [5]. Similar results were obtained in a study that validated DiroChek ${ }^{\circledR}$ in Brisbane, Queensland using a

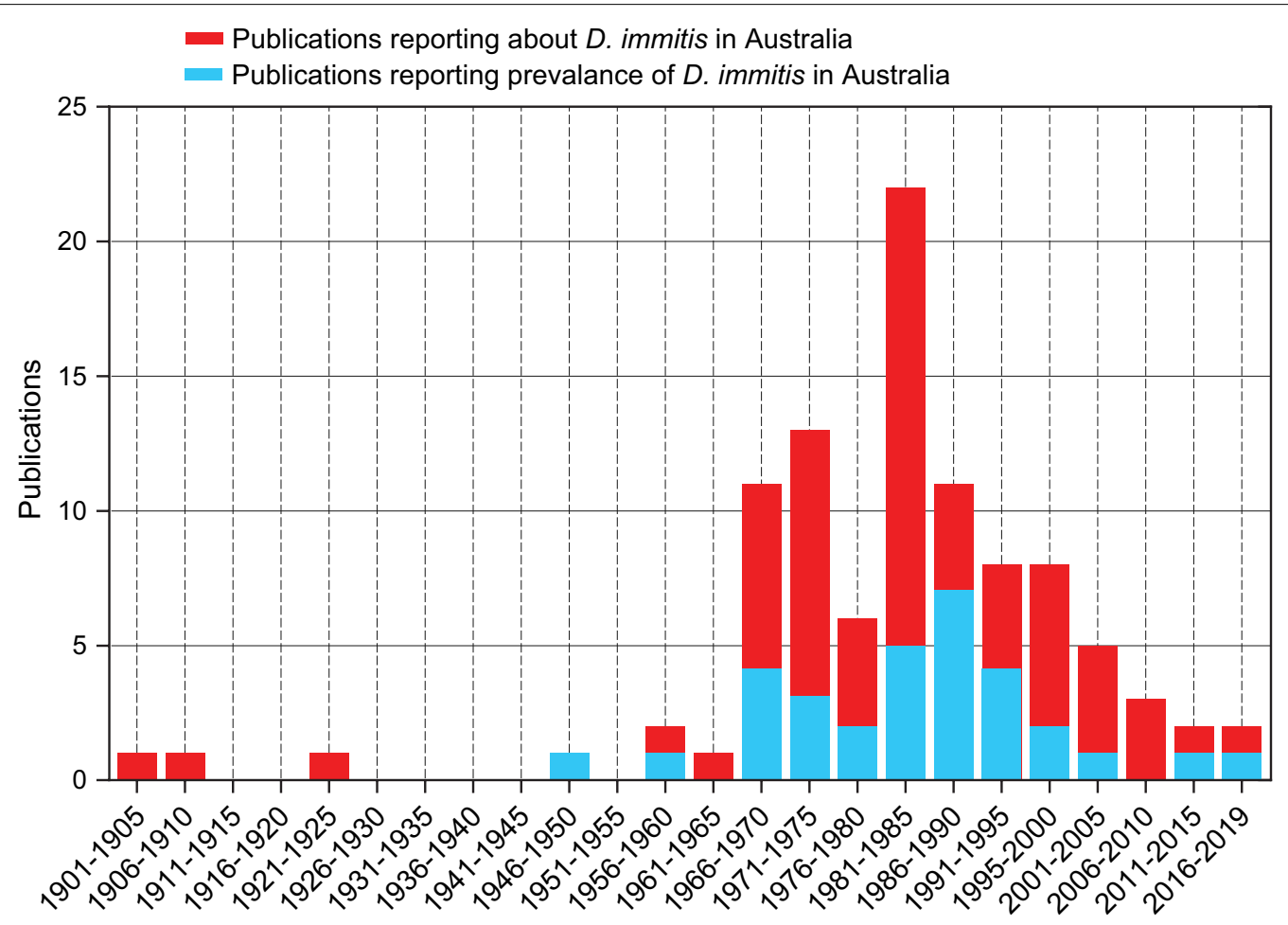

Fig. 3 Histogram of published studies on Dirofilaria immitis in Australia from 1901 to 2019. All articles were manually inspected to confirm the presence D. immitis data in Australia (red, $n=98$ ) and studies reporting prevalence data of D. immitis were identified (blue, $n=32$ ). Inclusion criteria for retained references included primary data on $D$. immitis prevalence in dogs, regardless of the method used 
Table 4 Summary of canine heartworm prevalence data in Australia from published studies between 1901-2019

\begin{tabular}{|c|c|c|c|c|c|c|}
\hline Location & Year(s) & Sample size & Sample source & Method of detection & Prevalence (\%) & Reference \\
\hline \multicolumn{7}{|l|}{ Queensland } \\
\hline Brisbane & 1959 & Unknown & Dogs & Necropsy & 10 (unspecified) & {$[37]$} \\
\hline Brisbane & 1964-1965 & 761 & RSPCA dogs & Necropsy and/or Mff & $\begin{array}{l}24(183 / 761 ; 95 \% \mathrm{Cl}: \\
21.1-27.2)\end{array}$ & {$[6]$} \\
\hline Brisbane & 1968 & 114 & Pound dogs & Unknown & 17 (unspecified) & {$[38]$} \\
\hline Townsville & 1969 & 94 & Vet clinic dogs & Mff & $\begin{array}{l}37(35 / 94 ; 95 \% \mathrm{Cl}: \\
28.1-47.3)\end{array}$ & [6] \\
\hline Brisbane & & 296 & & & $\begin{array}{l}21 \text { (61/296; } 95 \% \mathrm{Cl}: \\
16.4-25.6)\end{array}$ & \\
\hline Brisbane & 1969-1971 & 238 & Dogs & Mff & $\begin{array}{l}15 \text { (37/238; 95\% Cl: } \\
11.5-20.7)\end{array}$ & {$[10]$} \\
\hline Cherbourg & & 16 & Dogs & & $6(1 / 16 ; 95 \% \mathrm{Cl}:<0.1-30.3)$ & \\
\hline Edward River & & 36 & Dogs & & $\begin{array}{l}85 \text { (31/36; } 95 \% \mathrm{Cl}: \\
70.9-94.4)\end{array}$ & \\
\hline Aurukun & & 23 & Dogs & & $\begin{array}{l}90 \text { (20/23; 95\% Cl: } \\
67.0-96.3)\end{array}$ & \\
\hline Bamaga & & 48 & Dogs & & $\begin{array}{c}25(12 / 48 ; 95 \% \mathrm{Cl}: \\
14.8-38.9)\end{array}$ & \\
\hline Hope Vale & & 86 & Dogs & & 0 (0/86; 95\% Cl: 0-5.1) & \\
\hline Mornington Island & & 14 & Dogs & & 0 (0/14; 95\% Cl: 0-25.2) & \\
\hline Kowanyama & & 84 & Dogs & Necropsy & $\begin{array}{l}88 \text { (78/84; } 95 \% \mathrm{Cl}: \\
85.0-97.0)\end{array}$ & {$[10]$} \\
\hline Townsville & 1972 & 28 & Owned dogs & Mff & 68 (unspecified) & [39] \\
\hline Brisbane & 1972-1976 & 480 & Dogs & Mff & $\begin{array}{l}36 \text { (171/480; 95\% Cl: } \\
31.5-40.0)\end{array}$ & {$[10]$} \\
\hline Cherbourg & & 80 & Dogs & & 8 (6/80; 95\% Cl: 3.2-15.7) & \\
\hline Kowanyama & & 45 & Dogs & & $\begin{array}{l}64(29 / 45 ; 95 \% \mathrm{Cl}: \\
49.8-76.8)\end{array}$ & \\
\hline Edward River & & 23 & Dogs & & $\begin{array}{l}78 \text { (18/23; 95\% Cl: } \\
57.7-90.8)\end{array}$ & \\
\hline Bamaga & & 45 & Dogs & & $\begin{array}{l}71(32 / 45 ; 95 \% \mathrm{Cl}: \\
56.5-82.4)\end{array}$ & \\
\hline Brisbane & 1979 & 120 & Pound dogs & Mff & $\begin{array}{l}36 \text { (43/120; } 95 \% \text { Cl: } \\
27.8-44.7)\end{array}$ & [7] \\
\hline Brisbane & 1981 & 100 & Stray puppies (> 8 weeks) & Mff & 1 (1/100; 95\% Cl: <0.1-6.0) & {$[40]$} \\
\hline Brisbane & 1986 & 57 & Pound dogs & Necropsy & $\begin{array}{l}60(34 / 57 ; 95 \% \mathrm{Cl}: \\
46.7-71.4)\end{array}$ & [41] \\
\hline Brisbane & 1986 & 59 & Pound dogs & Necropsy & $\begin{array}{l}64(35 / 59 ; 95 \% \mathrm{Cl}: \\
46.6-70.9)\end{array}$ & [42] \\
\hline Brisbane & 1988 & 125 & Stray dogs & Necropsy ${ }^{a}$ & $\begin{array}{l}42(52 / 125 ; 95 \% \mathrm{Cl}: 33 . \\
\quad 3-50.7)\end{array}$ & {$[28]$} \\
\hline Brisbane & 1989 & 100 & Dogs & Necropsy & $\begin{array}{l}22 \text { (22/100; } 95 \% \mathrm{Cl}: \\
14.9-31.1)\end{array}$ & [12] \\
\hline Brisbane & 1991 & 272 & Pound dogs & Necropsy & $\begin{array}{l}49 \text { (134/272; } 95 \% \mathrm{Cl}: \\
\quad 43.4-55.2)\end{array}$ & {$[25]$} \\
\hline Brisbane & 1993 & 57 & Pound dogs & Necropsy & $\begin{array}{l}60(34 / 57 ; 95 \% \mathrm{Cl}: \\
46.7-71.4)\end{array}$ & [43] \\
\hline \multirow[t]{2}{*}{ Townsville } & 2001 & Unknown & Pound dogs & Unknown & 15 (unspecified) & [44] \\
\hline & 2002 & 27 & Wild dogs & & $\begin{array}{l}56(15 / 27 ; 95 \% \mathrm{Cl}: \\
37.3-72.4)\end{array}$ & \\
\hline Yarrabah & 2008-2012 & 51 & Remote community dogs & SNAP (Idexx) & $2(1 / 51 ; 95 \% \mathrm{Cl}:<0.1-11.3)$ & {$[45]$} \\
\hline Cairns & 2007-2013 & 23 & $\begin{array}{l}\text { Wild + urban fringe } \\
\text { dingoes }\end{array}$ & $\begin{array}{l}\text { Necropsy, Mff, SNAP } \\
\quad(\text { Idexx) }\end{array}$ & $\begin{array}{l}44 \text { (10/23; } 95 \% \mathrm{Cl}: \\
25.6-63.2)\end{array}$ & [19] \\
\hline Atherton & 2007-2013 & 5 & Urban fringe dingoes & Necropsy & 0 (0/5; 95\% Cl: 0-48.9) & [19] \\
\hline
\end{tabular}


Table 4 (continued)

\begin{tabular}{|c|c|c|c|c|c|c|}
\hline Location & Year(s) & Sample size & Sample source & Method of detection & Prevalence (\%) & Reference \\
\hline \multicolumn{7}{|l|}{ New South Wales } \\
\hline $\begin{array}{l}\text { Murrumbidgee Irrigation } \\
\text { Area (Southern NSW) }\end{array}$ & 1966 & Unknown & Necropsy dogs & Necropsy & 50 (unspecified) & {$[46]$} \\
\hline Sydney & 1968 & 62 & Dogs & Mff & $\begin{array}{l}19(12 / 62 ; 95 \% \mathrm{Cl}: \\
11.3-31.0)\end{array}$ & {$[47]$} \\
\hline Sydney & 1971-1972 & 495 & Pound dogs & Necropsy & 4 (21/495; 95\% Cl: 2.8-6.4) & {$[26]$} \\
\hline Sydney & 1971 & 40 & Vet clinic dogs & Mff & 8 (3/40; 95\% Cl: 1.9-20.6) & {$[26]$} \\
\hline Sydney & 1972 & 339 & Pound dogs & Necropsy & 3 (9/339; 95\% Cl: 1.3-5.0) & {$[48]$} \\
\hline $\begin{array}{l}\text { Hunter Valley/Coastal } \\
\text { NSW }\end{array}$ & 1979-1981 & 331 & Greyhounds & Mff & $\begin{array}{l}11 \text { (36/331; } 95 \% \mathrm{Cl}: \\
\quad 7.9-14.7)\end{array}$ & {$[9]$} \\
\hline Sydney & $1981-1983$ & 405 & Vet clinic dogs & Mff & $\begin{array}{l}13(51 / 405 ; 95 \% \mathrm{Cl} \\
\quad 9.7-16.2)\end{array}$ & {$[49]$} \\
\hline Sydney & 1982-1983 & 68 & Red foxes Vulpes vulpes & Necropsy & 9 (6/68; 95\% Cl: 3. 8-18.3) & {$[49]$} \\
\hline Sydney & 1983 & 100 & Pound dogs & Necropsy & $\begin{array}{l}50 \text { (50/100; } 95 \% \mathrm{Cl}: \\
40.4-59.6)\end{array}$ & {$[50]$} \\
\hline Sydney & 1987 & 100 & Pound dogs & Necropsy $^{a}$ & $\begin{array}{l}24 \text { (24/100; } 95 \% \mathrm{Cl}: \\
16.6-33.3)\end{array}$ & [29] \\
\hline Sydney & 1987 & 100 & Pound dogs & Necropsy & $\begin{array}{l}32(32 / 100 ; 95 \% \mathrm{Cl}: 23.7 \\
\quad \text { to } 41.7)\end{array}$ & {$[51]$} \\
\hline \multirow[t]{2}{*}{ Sydney } & 1992 & 304 & Pound dogs & Mffa $^{a}$ & $\begin{array}{l}12(36 / 304 ; 95 \% \mathrm{Cl}: \\
\quad 8.7-16.0)\end{array}$ & {$[5]$} \\
\hline & & 100 & & Necropsy $^{a}$ & $\begin{array}{l}15 \text { (15/100; } 95 \% \mathrm{Cl}: \\
9.2-23.4)\end{array}$ & \\
\hline Collarenebri & 2008-2009 & 39 & Remote community dogs & SNAP (Idexx) & 0 (0/39; 95\% Cl: 0-10.7) & {$[45]$} \\
\hline Goodooga & 2008-2009 & 19 & Remote community dogs & SNAP (Idexx) & 21 (4/19; 95\% Cl: 8.0-43.9) & {$[45]$} \\
\hline \multicolumn{7}{|l|}{ Victoria } \\
\hline Melbourne & 1946 & 174 & Ownerless dogs & Necropsy & 0 (0/174; 95\% Cl: 0-2.6) & {$[52]$} \\
\hline \multirow[t]{2}{*}{ North-eastern Victoria } & 1978 & 752 & Pound dogs (734) & Necropsy & 2 (15/734; 95\% Cl: 1.2-3.4) & {$[53]$} \\
\hline & & & Greyhounds (18) & & $\begin{array}{l}28(5 / 18 ; 95 \% \mathrm{Cl}: \\
12.2-51.2)\end{array}$ & \\
\hline Melbourne & 1991-1994 & 125 & Red foxes (Vulpes vulpes) & Dirochek $^{\circledR}$ (Symbiotics) & 6 (8/125; 95\% Cl: 3.1-12.3) & {$[54]$} \\
\hline \multicolumn{7}{|l|}{ Northern Territory } \\
\hline Darwin & 1963 & Unknown & Dogs & Unknown & 70 (unspecified) & {$[6]$} \\
\hline Alice Springs & 1972-1976 & 98 & Vet clinic dogs & Mff & $\begin{array}{l}13(12 / 98 ; 95 \% \mathrm{Cl}: \\
0.1-20.3)\end{array}$ & {$[10]$} \\
\hline $\begin{array}{l}\text { Docker River + Indul- } \\
\text { kana }\end{array}$ & & 73 & Remote community dogs & Mff & 0 (0/73; 95\% Cl: 0-6.0) & {$[10]$} \\
\hline Katherine & 1988 & 32 & Dingoes & Necropsy & $\begin{array}{l}56(18 / 32 ; 95 \% \mathrm{Cl}: \\
39.3-71.9)\end{array}$ & {$[55]$} \\
\hline TiTree & 2007-2009 & 48 & Remote community dogs & SNAP (Idexx) & 2.5 (4/48; 95\% Cl: 2.8-20.1) & {$[45]$} \\
\hline Tiwi Islands & 2008-2009 & 27 & Remote community dogs & SNAP (Idexx) & 0 (0/27; 95\% Cl: 0-14.8) & {$[45]$} \\
\hline \multicolumn{7}{|l|}{ South Australia } \\
\hline South Australia & 1988-1991 & 1658 & Dogs & Mff & $\begin{array}{l}1 \text { (19/1658; 95\% Cl: } \\
\quad 0.7-1.8)\end{array}$ & {$[8]$} \\
\hline \multicolumn{7}{|l|}{ Western Australia } \\
\hline Kimberly & 1993 & 58 & Remote community dogs & Necropsy & 0 (0/58; 95\% Cl: 0-7.4) & {$[56]$} \\
\hline Bidyadanga & 2008 & 15 & Remote community dogs & SNAP (Idexx) & 0 (0/15; 95\% Cl: 0-23.9) & {$[45]$} \\
\hline
\end{tabular}

a Validation of DiroChek ${ }^{\circledR}$ Symbiotics Corp. USA supplied by Commonwealth Serum Laboratories, Parkville, Victoria (now owned by Zoetis, NJ)

Abbreviations: $95 \% \mathrm{Cl}$, 95\% confidence interval; $\mathrm{Mff}$, detection of microfilariae

cohort of 125 necropsied dogs (52/125, 42\% infected with D. immitis) with a reported sensitivity and specificity of $73.1 \%$ and $95.9 \%$, respectively [28].
Henry et al. [22] demonstrated Mff absence in 19\% of the necropsy positive dogs with female $D$. immitis, nevertheless absence of Mff was reported in dogs even with 
burden of $>40$ female $D$. immitis $(18 \%, 4 / 50)$. In Australia, absence of Mff was reported in 65\% (15/26) and $20 \%(3 / 15)$ of $D$. immitis necropsy positive dogs from Brisbane [28] and Sydney [5], respectively. In our study, we applied species-specific PCR to detect the presence of microfilarial DNA, which was absent in four of DiroChek ${ }^{\circledR}$ antigen positive dogs. These findings confirm that detection of microfilariae by filtration (Difil) or concentration (Knotts) techniques are complimentary to DiroChek ${ }^{\circledR}$ to identify microfilariae-positive dogs prior to antigen testing and should be performed whenever screening at-risk dogs for $D$. immitis.

Detection of low burdens of $D$. immitis in dogs is difficult, because DiroChek ${ }^{\circledR}$ sensitivity can be variable (53\% in [28]; 62.5\% in [29]; 86\% in [27]) due to unknown worm burden distribution in the dog population. In areas of recent heartworm introduction, newly infected dogs will have no Mff due to the prepatent period of at least six months, single sex infections or burdens with less than two female heartworms. Additionally, the formation of immune complexes can further complicate diagnosis and detection of D. immitis antigen [30,31].

\section{Conclusions}

The fall in the prevalence of $D$. immitis in Australia since the introduction of MLs (monthly tablets and chews, top-spots and yearly depot injections) contrasts to the situation in most of the USA and Canada where the prevalence and extent of heartworm disease has increased despite the availability of these drugs [32-34]. In Australia, preventative drugs are widely available at veterinary clinics, pet stores, supermarkets and on the internet via online veterinary wholesalers, providing easy access to prophylactic therapy $[13,35]$. More restricted availability of MLs in North America, reduced competition in the marketplace and possibly higher cost (as a result) may in part explain why heartworm disease has been virtually eradicated from NSW in Australia, in contrast to most of the USA [36]. Additionally, NSW has been in extended drought conditions for three years, which presumably has reduced the availability of mosquito vectors. In contrast to NSW, where we did not demonstrate canine heartworm infection in dog cohorts receiving no or intermittent prevention, Queensland pig-hunting dogs are identified as the at-risk population with $21 \%$ D. immitis antigen prevalence in Central Queensland.

\section{Abbreviations \\ NDA: no detectable antigen; NSW: New South Wales; MLs: macrocyclic lac- tones; ELISA: enzyme-linked immunosorbent assay; Mff: microfilariae.}

\section{Acknowledgements}

We acknowledge the support provided by the Veterinary Pathology Diagnostics Services (VPDS) at The University of Sydney, RSPCA NSW, Greyhound
Adoption Program (NSW) Inc, Southern Tablelands Veterinary Hospital, Sarina Veterinary Surgery, Proserpine Veterinary Clinic, Gold City Veterinary Clinic, Clermont Veterinary Surgery, Tableland Veterinary Service, Tropical Vet Services and Dr Jackie Picard at James Cook University.

\section{Authors' contributions}

BO, JŠ, JN, GM, DW, RM and MEW designed the study. BO, GM, JŠ and DW collected samples and conducted antigen tests. JŠ, MW and JN supervised laboratory work and statistical analysis. WLK conducted the historical literature review. BO, JŠ, RM, MEW, JN, GB, GM, DW and MEW and WLK interpreted the results. All authors read and approved the final manuscript.

\section{Funding}

This work was supported by an Australian Companion Animal Health Foundation Research Grant 007/2019. BO is supported by an Australian Government Research Training Program scholarship and has received funding for research from the Marie-Bashir Institute Zoonoses Node. RM is supported by the Valentine Charlton Bequest. Funding bodies had no role in the design, analysis, collection of samples, or writing of the manuscript.

\section{Availability of data and materials}

The data analyzed in this article are included within the article.

\section{Ethics approval and consent to participate}

Human (2018/317) and animal ethics (2016/1044; 2018/1341; 2016/995) approval was obtained from The University of Sydney's Human Research Ethics Committee and Animal Ethics Committee, respectively.

\section{Consent for publication}

Not applicable.

\section{Competing interests}

The authors declare that they have no competing interests.

\section{Author details}

${ }^{1}$ Sydney School of Veterinary Science, The University of Sydney, Sydney, NSW 2006, Australia. ${ }^{2}$ Centre for Veterinary Education, The University of Sydney, Sydney, NSW 2006, Australia. ${ }^{3}$ Greyhound Adoption Program (NSW), PO Box 24, Belrose West, NSW 2085, Australia.

Received: 28 November 2019 Accepted: 4 February 2020

Published online: 13 February 2020

\section{References}

1. McCall JW, Genchi C, Kramer LH, Guerrero J, Venco L. Heartworm disease in animals and humans. Adv Parasitol. 2008:66:193-285.

2. Bowman DD. Heartworms, macrocyclic lactones, and the specter of resistance to prevention in the United States. Parasit Vectors. 2012;5:138.

3. Kramer L, Crosara S, Gnudi G, Genchi M, Mangia C, Viglietti A, et al. Wolbachia, doxycycline and macrocyclic lactones: new prospects in the treatment of canine heartworm disease. Vet Parasitol. 2018;254:95-7.

4. Polak KC, Smith-Blackmore M. Animal shelters: managing heartworms in resource-scarce environments. Vet Parasitol. 2014;206:78-82.

5. Bidgood A, Collins GH. The prevalence of Dirofilaria immitis in dogs in Sydney. Aus Vet J. 1996;73:103-4.

6. Carlisle $\mathrm{CH}$. The incidence of Dirofilaria immitis (heartworm) in dogs in Queensland. Aus Vet J. 1969;45:535-8.

7. Atwell RB, Carlisle C. Canine dirofilariasis in the metropolitan area of Brisbane. Aus Vet J. 1979;55:399.

8. Copland MD, O'Callaghan MG, Hajduk P, O'Donoghue PJ. The occurrence of Dirofilaria immitis in dogs in South Australia. Aus Vet J. 1992;69:31-2.

9. Martin TE, Collins GH. Prevalence of Dirofilaria immitis and Dipetalonema reconditum in greyhounds. Aus Vet J. 1985;62:159-63.

10. Welch JS, Dobson C, Freeman C. Distribution and diagnosis of dirofilariasis and toxocariasis in Australia. Aus Vet J. 1979;55:265-74.

11. Doyle K. Heartworm treatment-NRA decision opposed. Aus Vet J. 2001;72:74 
12. Tarish $J H$, Atwell RB. Evaluation of protein-A gold labelling with Dirofilaria immitis antigen coated nitrocellulose for the detection of anti-heartworm antibody in dogs. Aus Vet J. 1989;66:295.

13. Nguyen C, Koh WL, Casteriano A, Beijerink N, Godfrey C, Brown G, et al. Mosquito-borne heartworm Dirofilaria immitis in dogs from Australia. Parasit Vectors. 2016;9:535.

14. Russell RC, Geary MJ. The influence of microfilarial density of dog heartworm Dirofilaria immitis on infection rate and survival of Aedes notoscriptus and Culex annulirostris from Australia. Med Vet Entomol. 1996;10:29-34.

15. Bemrick WJ, Moorhouse DE. Potential vectors of Dirofilaria immitis in the Brisbane area of Queensland, Australia. J Med Entomol. 1968;5:269-72.

16. Wolstenholme AJ, Evans CC, Jimenez PD, Moorhead AR. The emergence of macrocyclic lactone resistance in the canine heartworm, Dirofilaria immitis. Parasitology. 2015;142:1249-59.

17. Drake J, Wiseman S. Increasing incidence of Dirofilaria immitis in dogs in USA with focus on the southeast region 2013-2016. Parasit Vectors. 2018;11:39.

18. Genchi C, Bowman D, Drake J. Canine heartworm disease (Dirofilaria immitis) in western Europe: survey of veterinary awareness and perceptions. Parasit Vectors. 2014;7:206.

19. Smout FA, Skerratt LF, Butler JR, Johnson CN, Congdon BC. Dingoes (Canis dingo Meyer, 1793) continue to be an important reservoir host of Dirofilaria immitis in low density housing areas in Australia. Vet Parasitol. 2016;215:6-10.

20. Orr B, Malik R, Norris J, Westman M. The welfare of pig-hunting dogs in Australia. Animals (Basel). 2019;9:853. https://doi.org/10.3390/ani9100853.

21. Hunt H, Cave NJ, Gartrell BD, Cogger N, Petersen JA, Roe WD. An epidemiological investigation of an idiopathic myopathy in hunting dogs in New Zealand. N Z Vet J. 2018;66:199-204.

22. Henry LG, Brunson KJ, Walden HS, Wenzlow N, Beachboard SE, Barr KL et al. Comparison of six commercial antigen kits for detection of Dirofilaria immitis infections in canines with necropsy-confirmed heartworm status. Vet Parasitol. 2018;254:178-82.

23. Lefever S, Vandesompele J, Speleman F, Pattyn F. RTPrimerDB: the portal for real-time PCR primers and probes. Nucleic Acids Res. 2009;37(Database issue):D942-5.

24. Rishniw M, Barr S, Simpson K, Frongillo M, Franz M, Dominguez Alpizar $J$. Discrimination between six species of canine microfilariae by a single polymerase chain reaction. Vet Parasitol. 2006:135:303-14.

25. Tanaka K, Atwell RB. The humoral response in natural Dirofilaria immitis infections in dogs. Aus Vet J. 1991;68:396-9.

26. Watson AD, Porges WL, Testoni FJ. A survey of canine filariasis in Sydney. Aus Vet J. 1973:49:31-4

27. Courtney $\mathrm{CH}$, Cornell JA. Evaluation of heartworm immunodiagnostic tests. J Am Vet Med Assoc. 1990;197:724-9.

28. Atwell RB, Sheridan AB, Baldock FC. An evaluation of the Dirochek test for detection of Dirofilaria immitis antigen in dogs. Aus Vet J. 1988;65:161.

29. Collins GH, Pope SE. An evaluation of an ELISA test for the detection of antigens of Dirofilaria immitis. Aus Vet J. 1987;64:318-9.

30. Drake J, Gruntmeir J, Merritt H, Allen L, Little SE. False negative antigen tests in dogs infected with heartworm and placed on macrocyclic lactone preventives. Parasit Vectors. 2015;8:68

31. Little $S$, Saleh M, Wohltjen M, Nagamori Y. Prime detection of Dirofilaria immitis: understanding the influence of blocked antigen on heartworm test performance. Parasit Vectors. 2018;11:186.

32. Bowman DD, Drake J. Examination of the "susceptibility gap" in the treatment of canine heartworm infection. Parasit Vectors. 2017;10(Suppl. 2):513.

33. McCall JW. The safety-net story about macrocyclic lactone heartworm preventives: a review, an update, and recommendations. Vet Parasitol. 2005:133:197-206.

34. Ballesteros C, Pulaski CN, Bourguinat C, Keller K, Prichard RK, Geary TG. Clinical validation of molecular markers of macrocyclic lactone resistance in Dirofilaria immitis. Int J Parasitol Drugs Drug Resist. 2018;8:596-606.
35. Dearsley EJ, O'Handley RM, Caraguel C. Is canine heartworm (Dirofilaria immitis) endemic to South Australia? Aust Vet J. 2019;97:191-6.

36. Self SW, Pulaski CN, McMahan CS, Brown DA, Yabsley MJ, Gettings $J R$. Regional and local temporal trends in the prevalence of canine heartworm infection in the contiguous United States: 2012-2018. Parasit Vectors. 2019;12:380.

37. Winter $\mathrm{H}$. The pathology of canine dirofilariasis. Am J Vet Res. 1959;20:366-71.

38. Roy-Smith F, Carlisle $\mathrm{CH}$. A field study of chemical prophylaxis against the developing stages of Dirofilaria immitis in the dog. Aus Vet J. 1968:44:508-9.

39. Aubrey JN, Copeman DB. Canine dirofilarosis - an evaluation of bimonthly diethylcarbamazine therapy in prophylaxis. Aus Vet J. 1972;48:310-2.

40. Atwell RB. Prevalence of Dirofilaria immitis microfilariaemia in 6- to 8-week-old pups. Aus Vet J. 1981;57:479.

41. Atwell RB, Rezakhani A. The effectiveness of a latex agglutination test for the detection of circulating antigen of Dirofilaria immitis in dogs. Aus Vet J. 1986;63:130-1.

42. Atwell RB, Rezakhani A. Preliminary assessment of Dirocult, a latex agglutination test for detection of Dirofilaria immitis infection in the dog. Aus Vet J. 1986;63:127

43. Tanaka Kl, Atwell RB. Immunohistological observations on pulmonary tissues from dogs infected with Dirofilaria immitis. Vet Res Commun. 1993:17:109.

44. Brown B, Copeman DB. Zoonotic importance of parasites in wild dogs caught in the vicinity of Townsville. Aus Vet J. 2003;81:700-2.

45. Šlapeta J, Dowd SE, Alanazi AD, Westman ME, Brown GK. Differences in the faecal microbiome of non-diarrhoeic clinically healthy dogs and cats associated with Giardia duodenalis infection: impact of hookworms and coccidia. Int J Parasitol. 2015;45:585-94.

46. Rees D, Sharrock AG, Lillecrap JA. Canine dirofilariasis in the Murrumbidgee irrigation area. Aus Vet J. 1966;42:61-2.

47. Whitlock LE. The incidence of microfilaria in canine blood in Sydney. Aus Vet J. 1969:45:136

48. Holmes PR, Kelly JD. The incidence of Dirofilaria immitis and Dipetalonema reconditum in dogs and cats in Sydney. Aus Vet J. 1973;49:55.

49. Mulley RC, Starr TW. Dirofilaria immitis in red foxes (Vulpes vulpes) in an endemic area near Sydney, Australia. J Wildl Dis. 1984;20:152-3.

50. Martin TE, Collins GH, Griffin DL, Pope SE. An evaluation of 4 commercially available ELISA kits for the diagnosis of Dirofilaria immitis infection in dogs. Aus Vet J. 1985;62:166-9.

51. Collins GH, Griffin DL, Pope SE. Evaluation of a latex agglutination test for the diagnosis of infection with Dirofilaria immitis in dogs. Aus Vet J. 1987;64:317-8

52. Pullar EM. A survey of Victorian canine and vulpine parasites: IV, Nematoda. Aus Vet J. 1946:22:85-91.

53. Blake RT, Overend DJ. The prevalence of Dirofilaria immitis and other parasites in urban pound dogs in north-eastern Victoria. Aus Vet J. 1982:58:111-4

54. Marks CA, Bloomfield TE. Canine heartworm (Dirofilaria immitis) detected in red foxes (Vulpes vulpes) in urban Melbourne. Vet Parasitol. 1998;78:147-54.

55. Starr TW, Mulley RC. Dirofilaria immitis in the dingo (Canis familiaris dingo) in a tropical region of the Northern Territory, Australia. J Wildl Dis. 1988:24:164-5.

56. Thompson RC, Meloni BP, Hopkins RM, Deplazes P, Reynoldson JA. Observations on the endo- and ectoparasites affecting dogs and cats in aboriginal communities in the north-west of Western Australia. Aus Vet J. 1993;70:268-70.

\section{Publisher's Note}

Springer Nature remains neutral with regard to jurisdictional claims in published maps and institutional affiliations. 